\title{
Akımsız Ni-P-W Kompozit Kaplamalarda PTFE Konsantrasyonunun Sertlik ve Aşınma Üzerinde Etkisi
}

\author{
Serdar Aslan ${ }^{1 *}$, Erhan Duru ${ }^{2}$ \\ 1* Sakarya Üniversitesi, Mühendislik Fakültesi, Metalurji ve Malzeme Mühendisliği Bölümü, Sakarya, Türkiye, (ORCID: 0000-0001-5061-6338), \\ saslan@sakarya.edu.tr \\ ${ }^{2}$ Sakarya Üniversitesi, Mühendislik Fakültesi, Metalurji ve Malzeme Mühendisliği Bölümü, Sakarya, Türkiye, (ORCID: 0000-0002-6205-6566), \\ erhanduru@sakarya.edu.tr
}

(1st International Conference on Applied Engineering and Natural Sciences ICAENS 2021, November 1-3, 2021)

(DOI: 10.31590/ejosat.1016986)

ATIF/REFERENCE: Aslan, S. \& Duru, E. (2021). Akımsız Ni-P-W Kompozit Kaplamalarda PTFE Konsantrasyonunun Sertlik ve Aşınma Üzerinde Etkisi. Avrupa Bilim ve Teknoloji Dergisi, (28), 1356-1359.

$\ddot{O} \mathbf{z}$

Bu çalışmada politetrafloroetilen (PTFE) takviyeli Ni-P-W kompozit kaplamalar alkalin bir banyoda akımsız yöntemle üretilmiştir. Kompozit kaplamalar için PTFE konsantrasyonu sırası ile 5, 10 ve 20 g/L seçilmiştir. Üretilen kaplamaların yüzey mikroyapısı Taramalı Elektron Mikroskobu (SEM) ile kimyasal analizi ise X-Işınları Kırınımı (XRD) yöntemiyle yapılmıştır. Farklı PTFE konsantrasyonları ile üretilen kompozit kaplamaların ortalama mikro sertlik değerleri sırasılla $513 \pm 22$ HV, 494 \pm 17 HV ve $392 \pm 25$ HV olarak ölçülmüştür. Oda sıcaklığında kuru ortamda yapılan aşınmalarda en düşük sürtünme katsayısı $0.232 \mu$ ile PTFE konsantrasyonun 10 g/L olduğu kaplamada görülmüştür. Konsantrasyonun $20 \mathrm{~g} / \mathrm{L}$ çıkarılmasıyla ortalama sürtünme katsayısında yaklaşık \% 60 oranında bir artışla $0.375 \mu$ olmuştur.

\section{Effect of PTFE Concentration on Microhardness and Wear Behavior in Ni-P-W-PTFE Composite Coatings by Electroless Deposition}

\begin{abstract}
In this study, polytetrafluoroethylene (PTFE) reinforced Ni-P-W composite coatings were produced by electroless method in an alkaline bath. For the composite coatings, the PTFE concentration was chosen as 5, 10 and $20 \mathrm{~g} / \mathrm{L}$, respectively. The surface microstructure of the produced coatings was made by Scanning Electron Microscope (SEM) and chemical analysis was done by X-Ray Diffraction (XRD) method. The mean microhardness values of the composite coatings produced with different PTFE concentrations were measured as $513 \pm 22 \mathrm{HV}, 494 \pm 17 \mathrm{HV}$ and $392 \pm 25 \mathrm{HV}$, respectively. The lowest coefficient of friction in wear at room temperature was found to be $0.232 \mu$ in the coating with a PTFE concentration of $10 \mathrm{~g} / \mathrm{L}$. By increasing the concentration by $20 \mathrm{~g} / \mathrm{L}$, the average friction coefficient increased by approximately $60 \%$ to $0.375 \mu$.
\end{abstract}

Keywords: Electroless Coating, PTFE, Tungsten, Wear, Hardness.

\footnotetext{
*Sorumlu Yazar: $\underline{\text { saslan@ } @ \text { sakarya.edu.tr }}$
} 


\section{Giriş}

Son yüzyıldaki teknolojik gelişmeler üstün fiziksel ve kimyasal özelliklere sahip mühendislik malzemelerin üretiminde büyük gelişmelere yol açmıştır. Ancak malzemelerin fiziksel ve kimyasal özelliklerindeki bu büyük gelişmeler yine de malzemelerdeki aşınma ve korozyondan kaynaklı kayıpları engelleyememektedir. Günümüzde malzemeleri aşınma ve korozyondan korumak için pek çok yöntem bulunmaktadır. $\mathrm{Bu}$ yöntemlerden biri de malzeme yüzeyine kaplama yapmaktır. Kaplama, malzeme ile ortam arasında bir bariyer görevi görerek malzemeyi dış etkenlerden muhafaza etmektedir.

Günümüzde çok fazla kaplama yöntemi bulunmaktadır ancak hem korozyon direnci hem de aşınma dirençlerinin yüksek olmasından dolayı akımsız kaplamalar en popüler kaplamalar arasında yerini almaktadır. Akımsız kaplama yöntemi ilk olarak Brenner ve Ridell'in 1946'daki deneyleri sonucunda ortaya çıkmıştır (Vitry \& Delaunois, 2015). Bu yöntemde; dışardan bir elektrik kaynağına ihtiyaç duyulmadan elektrolit içerisindeki Ni iyonları otokatalitik kimyasal indirgenme yöntemi ile malzeme yüzeyinde biriktirilmektedir. (A. Akyol et al., 2018; Czagány et al., 2017). İndirgenme reaksiyonlarının oluşması için gereken akım kaynağı, elektrolite ilave edilen indirgeyici ile sağlanır. Akımsız kaplama yönteminde kullanılan indirgeyici türü aynı zamanda kaplamanın kimyasal içeriğini de belirlemektedir. Nikel kaplamada indirgeyici olarak hidrazin (Haag et al., 2006), Ni-P kaplamada sodyum hipofosfit (Abdülkadir Akyol et al., 2019), Ni-B kaplamada ise dimetilaminoboran (DURU et al., 2021) veya sodyum borhidrür (Sürdem et al., 2019) kullanılır.

Akımsız nikel kaplamaların fiziksel ve kimyasal özelliklerini $\mathrm{P}, \mathrm{B}, \mathrm{Mo}, \mathrm{Cu}, \mathrm{W}$ vb. elementlerin takviyesi ile geliştirmek mümkündür (Balaraju \& Rajam, 2005; FENG et al., 2021; Yildiz et al., 2021). Bunlar arasindan P ilavesi yapılan Ni-P kaplamaların yapıdaki amorfluktan kaynaklı korozyon dirençlerinin yüksek olduğu iyi bilinmektedir. Ni-P kaplamaların korozyon direncinin yanında mekanik ve fiziksel özelliklerini geliştirmek için tungsten (W) ilavesi iyi bir tercihtir. Tungstenin yüksek erime noktası, yüksek çekme dayanımı ve yüksek sertlik gibi fiziksel özellikleri akımsız kaplamaların gelişmesine büyük katkı sunmuştur. $\mathrm{Bu}$ sayede Ni-P-W kaplamalar yüksek sertliklere, yüksek sıcaklık dayanımlarına ve yüksek termal kararlıklara sahip olmaktadır. (A. Akyol et al., 2018; Luo et al., 2018). Akımsız yöntem ile elde edilen kaplamaların yüksek korozyon ve aşınma dayanımları gibi fiziksel ve kimyasal özelliklerinin yanında karmaşı parçaların dahi homojen kaplaması ve kaplama kalınlığının kontrol edilmesi bu yöntemi daha da değerli kılmaktadır. Bu özellikleri sayesinde akımsız kaplamalar otomotivden havacılık endüstrisine, bilgisayardan elektronik parçalara, kimyadan tekstil sanayisine kadar birçok alanda uygulama imkânı bulmaktadır (Matik \& Citak, 2011; Vitry \& Delaunois, 2015).

$\mathrm{Bu}$ çalışmada akımsız kaplama yöntemiyle PTFE (politetrafloroetilen) takviyeli Ni-P-W kompozit kaplamalar üretilmiştir. PTFE'nin katı yağlayıcı özelliği ve kimyasal kararlığı gibi özellikleri sayesinde Ni-P-W kaplamaların mekanik ve kimyasal kararlığ 1 da artacaktır. PTFE'nin bu özellikleri sayesinde kendinden yağlayıcı, yapışma önleyici, düşük sürtünme katsayılı, yüksek aşınma ve korozyon dayanımına sahip kompozit kaplamalar üretilecektir (Ger \& Hwang, 2002; Huang et al., 2003;
Ramalho \& Miranda, 2005). Bu kaplamalar yüksek aşınma ve korozyon dayanımı gerektiren otomotiv, uzay ve havacılık, matbacılık, elektronik, tekstil ve petrokimya gibi pek çok endüstriyel alanda malzemelerin kullanım ömrünü arttıracaktır.

\section{Materyal ve Metot}

$\mathrm{Bu}$ çalışmada altlık malzeme olarak $30 \times 30 \times 5 \mathrm{~mm}$ ölçülerinde st 37 çeliği kullanılmıştır. Altlık malzeme, kaplama öncesi sırasıyla 120, 240, 400, 800 ve 1200 kumluk SiC zımpara kâğıtları ile zımparalanmıştır. Zımpara işleminden sonra yüzeydeki yağ ve benzeri kirliliklerden arındırmak için aseton ile durulanmıştır. Son olarak altlık kaplama banyosuna daldırılmadan önce hacimce \% 10'luk nitrik asit çözeltisinde dağlanarak yüzey aktif hale getirilmiştir. Kaplama banyosunda nikel iyon kaynağ olarak nikel sülfat, tungsten kaynağı olarak sodyum tungstat, fosfor kaynağı ve indirgeyici olarak sodyum hipofosfit kullanılmıştır. Sodyum sitratın banyodaki görevi kompleks oluşturucu, amonyum klorürün reaksiyon hızlandırıcı ve sitrik asidin görevi de kompoleks yapıcıdır. Kompozit kaplamalar üç farklı PTFE konsantrasyonu ile üretilmiştir. Kaplama banyosunun bileşenleri ve deney şartları Tablo1.'de verilmiştir.

Tablo 1. Kaplama banyosunun kimyasal bileşenleri ve çalışma koşulları.

\begin{tabular}{|c|c|c|c|}
\hline Banyo Bileșeni & $\operatorname{Miktar}(\mathrm{g} / \mathrm{L})$ & \multicolumn{2}{|l|}{ Şartlar } \\
\hline Nikel Sülfat & 200 & Sicaklık & $90^{\circ} \mathrm{C}$ \\
\hline Sodyum Sitrat & 15 & $p H$ & 8,5 \\
\hline Sodyum Tungstat & 60 & Kar. $\mathrm{Hlzl}$ & $300 \mathrm{rpm}$ \\
\hline Sodyum Hipofosfit & 4.4 & Süre & $60 d k$ \\
\hline Amonyum Klorür & 15 & & \\
\hline Sitrik Asit & 8 & & \\
\hline PTFE & $5,10,20$ & & \\
\hline
\end{tabular}

Ni-P-W-PTFE kompozit kaplamaların yüzey morfolojisi Taramalı Elektron Mikroskobu (SEM) ile görüntülenmiş ve element analizi için Enerji Dağıtıcı Spektroskopisi (EDS) kullanılmıştır. Kompozit kaplamalardaki fazları belirlemek için X-Işını Kırınımı (XRD) yöntemi tercih edilmiştir. XRD analizi $10-80^{\circ}$ arasında, $2 \% \mathrm{dk}$ tarama hızında, 1.54 A dalga boyuna sahip $\mathrm{Cu} \mathrm{Ka}$ radyasyonu kullanılarak yapılmıştır. Kaplamaların sertlikleri mikrosertlik yöntemlerinden biri olan Vickers sertlik yöntemi ile yapılmıştır. Sertlik ölçümünde 50 gram yük 10 saniye boyunca uygulanarak her numuneden 5 adet sertlik alınmış ve ortalaması hesaplanmıştır. Kompozit kaplamaların aşınma deneyleri oda sıcaklığında ball on disk yöntemi ile yapılmıştır. Aşındırıcı malzeme olarak $10 \mathrm{~mm}$ çapında alümina bilya kullanılmıştır. Aşınma için tercih edilen yük 3N, kayma hızı 30 $\mathrm{cm} / \mathrm{s}$ ve kayma mesafesi 300 metredir. Aşınma sonrası yüzeylerin incelenmesi yine SEM ile yapılmıştır 


\section{Araştırma Sonuçları ve Tartışma}

Farklı konsantrasyonlarda PTFE takviyesi ile üretilen Ni-PW-PTFE kompozit kaplamaların yüzey görüntüleri Şekil 1'de verilmiştir. Şekil 1'de görüldüğü gibi Ni-P-W kaplamalar nodüler yapıda oluşmuştur, şekillerde görülen siyah bölgeler ise PTFE'nin yüzeydeki görüntüsüdür. PTFE konsantrasyonu arttıkça kaplamadaki PTFE miktarı da artmıştır. 5 g/L PTFE konsantrasyonlu kaplamada ( Şekil 1(a)) PTFE tozları seyrek bir şekilde yüzeyde dağılmıştır. Toz konsantrasyonu $10 \mathrm{~g} / \mathrm{L}$ olduğunda yapıdaki PTFE tozları yüzeyde her yere homojen şekilde dağılmış hale gelmiştir (Şekil 1(b)). Ancak PTFE konsantrasyonu $20 \mathrm{~g} / \mathrm{L}$ çıkarıldı ğında ise nikel matrisin içerisinde PTFE tozların homojen dağılmadığı, tozların topaklandığ görülmüştür ( Şekil 1(c)). Ayrıca matris içerisine giren en fazla PTFE toz miktar1, 10 g/L konsantrasyonda elde edilen kaplamada elde edilmiştir.

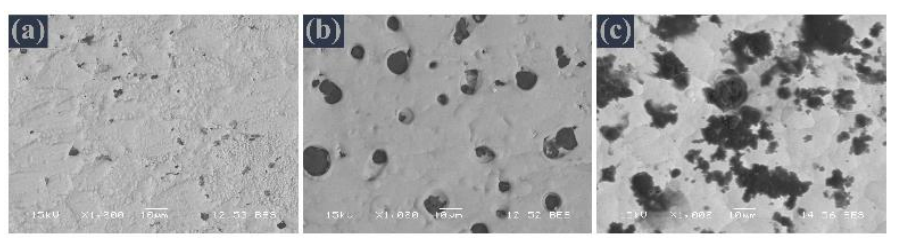

Şekil 1 Farklı konsantrasyonlarda PTFE takviyeli Ni-P-WPTFE kompozit kaplamalar: (a) $5 \mathrm{~g} / \mathrm{L}$ PTFE takviyeli, (b) $10 \mathrm{~g} / \mathrm{L}$ PTFE takviyeli ve (c) $20 \mathrm{~g} / \mathrm{L}$ PTFE takviyeli.

Farklı PTFE konsantrasyonlarında üretilen Ni-P-W-PTFE kompozit kaplamaların XRD analiz sonuçları Şekil 2'de verilmiştir. XRD piklerine bakıldığında Nikelin (111) düzlemine ait $2 \Theta=44^{\circ},(200)$ düzlemine ait $2 \Theta=52^{\circ}$ ve (220) düzlemine ait $2 \Theta=78^{\circ}$ pikleri görülmektedir. Her üç kaplamada yapı amorf olsa da PTFE miktarının artmasıyla yapıda amorfluk miktarı azalmıştır. PTFE tozlarının kaplamada Nikelin büyüme düzlemlerine etkisi olmuştur. Takviye edilen tozlar çekirdekleyici görevi görerek Nikelin (111) düzleminde tercihli yönlenmesine sebep olurken (200) ve (220) düzlemlerinin büyümesine engel olduğu görülmüştür (Doğan et al., 2020; Ürdem et al., 2021).

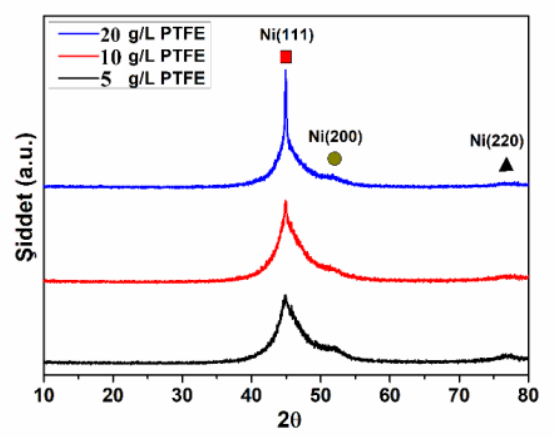

\section{Şekil 2 Ni-P-W-PTFE kompozit kaplamaların XRD analizi} sonucu.

Akımsız kompozit kaplamaların sertlikleri Şekil 3'de verilmiştir. PTFE konsantrasyonu arttıkça kompozit kaplamaların sertliğinde düşüş görülmüştür. $5 \mathrm{~g} / \mathrm{L}, 10 \mathrm{~g} / \mathrm{L}$ ve $20 \mathrm{~g} / \mathrm{L}$ PTFE konsantrasyonlu kaplamanın ortalama sertlikleri sırasıyla $513 \pm 22$ $\mathrm{HV}, 494 \pm 17 \mathrm{HV}$ ve $392 \pm 25 \mathrm{HV}$ çıkmıştır. $20 \mathrm{~g} / \mathrm{L}$ PTFE konsantrasyonlu kaplamada keskin düşüşün olmasının nedeni, kaplama içerisinde tozların topaklanması ve topaklanan tozlar nedeniyle meydana gelen porozite olduğu düşünülmektedir(Gültekin et al., 2021).

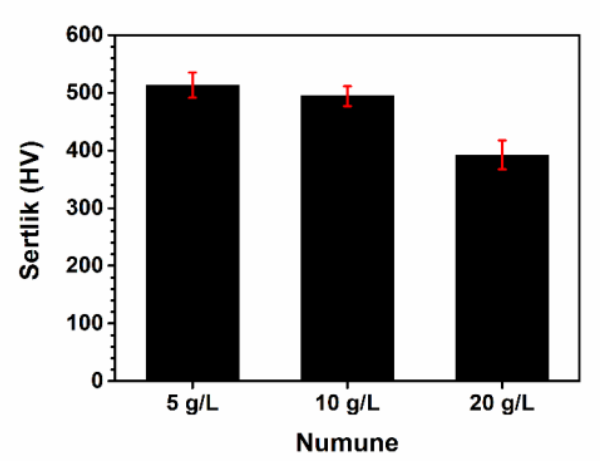

Şekil 3 Ni-P-W-PTFE kompozit kaplamaların ortalama sertlik sonuçları.

Kompozit kaplamaların aşınma sonrası ortalama sürtünme katsayısı ve aşınma oranı grafikleri Şekil 4'de verilmiştir. Toz konsantrasyonun $10 \mathrm{~g} / \mathrm{L}$ olduğu kaplamada ortalama sürtünme katsayısı $0.232 \mu$ ile en düşük sürtünme katsayısı elde edilmiştir. Nikel matrisin içerisine homojen bir şekilde giren PTFE tozlarının katı yağlayıcı özelliği sayesinde kaplamanın sürtünme katsayısı en düşük çıkmıştır. Konsantrasyonun $20 \mathrm{~g} / \mathrm{L}$ olduğu kaplamada ise tozların topaklanması sonucu $0.375 \mu$ 'luk ortalama sürtünme katsayısı ile en yüksek sürtünme katsayısı elde edilmiştir. Şekil 4(a)'da aşınma oranı ve aşınma iz genişliklerine bakıldığında yine paralel sonuçlar görülmüştür(Uysal et al., 2021).
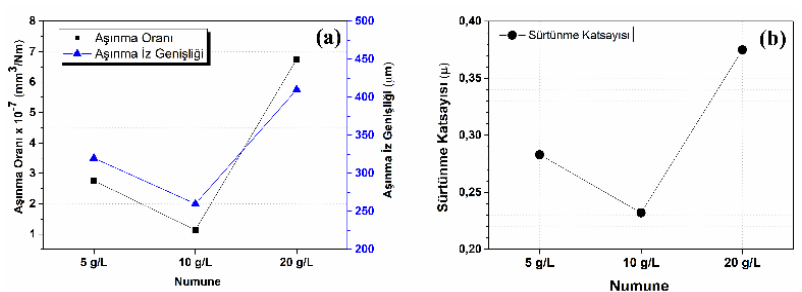

Şekil 4 Ni-P-W-PTFE kompozit kaplamaların aşınma oranı (a) ve ortalama sürtünme katsayısı (b) grafikleri.

Şekil 5'de kompozit kaplamaların aşınma sonrası yüzey SEM görüntüleri verilmiştir. $5 \mathrm{~g} / \mathrm{L}$ PTFE takviyeli kaplamada yüzeyde çatlaklar ve plastik deformasyon çizgileri meydana gelmiştir (Şekil 5(a)). Burada PTFE miktarı yetersiz kalmış ve kaplama yüzeyinde kısmen de olsa plastik deformasyon meydana geldiği görülmüştür. Şekil 5(b) ise $10 \mathrm{~g} / \mathrm{L}$ konsantrasyonlu kaplamanın aşınma yüzeyi görülmektedir. Toz konsantrasyonu $10 \mathrm{~g} / \mathrm{L}$ 'ye çıkarıldığında aşınma esnasında tozlar yüzeye sıvanarak yüzeyi aşınmaya karşı koruduğu görülmüştür. PTFE'nin kendinden yağlayıcı özelliği ve tozların yüzeye homojen dağılması sayesinde pürüzsüz ve çatlaksız bir yüzey oluşmuştur. Ancak toz konsantrasyonunun $20 \mathrm{~g} / \mathrm{L}$ çıkarılmasıyla kaplamada daha yıkıcı bir aşınma mekanizması ortaya çıkmıştır. PTFE tozların topaklanması ve yüzeye homojen dağılmaması deleminasyonlara yol açmış, aşırı çatlaklı bir yüzeyin oluşmasına yol açmıştır(Aslan \& Duru, 2021; DOĞAN et al., 2020; Uysal et al., 2021).
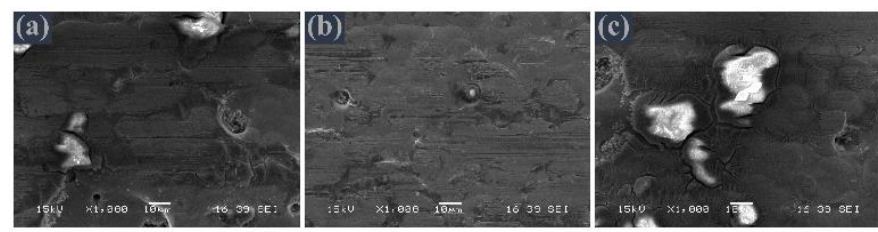

Şekil 5 Farklı konsantrasyonlarda PTFE takviyeli Ni-P-W-

PTFE kompozit kaplamaların aşınma sonrası yüzey SEM görüntüleri: (a) $5 \mathrm{~g} / \mathrm{L}$ PTFE takviyeli, (b) $10 \mathrm{~g} / \mathrm{L}$ PTFE takviyeli ve (c) $20 \mathrm{~g} / \mathrm{L}$ PTFE takviyeli. 


\section{Sonuç}

Ni-P-W-PTFE kompozit kaplamalar başarılı bir şekilde üretilmiştir. Kompozit kaplamalara uygulanan testlerin sonuçları aşağıda maddeler halinde verilmiştir.

PTFE konsantrasyonun $10 \mathrm{~g} / \mathrm{L}$ olduğu kaplamada, PTFE tozları matris içerisinde homojen dağılmıştır. Kaplamalar bu açıdan değerlendirildiğinde en iyi sonuç $10 \mathrm{~g} / \mathrm{L}$ PTFE konsantrasyonunda görülmüştür.

PTFE konsantrasyonu arttıkça kaplamanın sertliğinde bir düşüş görülmüştür.

Kompozit kaplamalar sürtünme katsayısı ve aşınma oranına göre karşılaştırıldığında en düşük değerler $10 \mathrm{~g} / \mathrm{L}$ PTFE konsantrasyonlu kaplamada görülmüş̧ür.

\section{Teşekkür}

Sakarya Üniversitesi Araştırma Geliştirme Merkezine (SARGEM) bize laboratuvar imkanlarını açtığı için teşekkür ederiz.

\section{Kaynakça}

Akyol, A., Algul, H., Uysal, M., Akbulut, H., \& Alp, A. (2018). A novel approach for wear and corrosion resistance in the electroless Ni-P-W alloy with CNFs co-depositions. Applied Surface Science, 453(December 2017), 482-492. https://doi.org/10.1016/j.apsusc.2018.05.152

Akyol, Abdülkadir, Algül, H., Bilaç, O., Ulu, S., Gül, H., Uysal, M., Çay, Y., \& Alp, A. (2019). Elektrolitik Sert Metal Kaplamaya Alternatif Çevreye Duyarlı Ni-P Kaplamaların 6061 Serisi Alüminyum Altlık Üzerine Akımsız Yöntemle Biriktirilmesinde Sodyum hipofosfit, Sicaklık ve Zamanın Etkisi. Academic Platform Journal of Engineering and Science, $7(3)$ 442-448. https://doi.org/10.21541/apjes.478232

Aslan, S., \& Duru, E. (2021). Microstructure and Wear Properties of Electrodeposited Ni-B-Al2O3 Composite Coating on Low Carbon Steel at Elevated Temperature. Journal of Materials Engineering and Performance, Ref 8. https://doi.org/10.1007/s11665-021-06290-2

Balaraju, J. N., \& Rajam, K. S. (2005). Electroless deposition of $\mathrm{Ni}-\mathrm{Cu}-\mathrm{P}, \mathrm{Ni}-\mathrm{W}-\mathrm{P}$ and $\mathrm{Ni}-\mathrm{W}-\mathrm{Cu}-\mathrm{P}$ alloys. Surface and Coatings Technology, 195(2-3), 154-161. https://doi.org/10.1016/j.surfcoat.2004.07.068

Czagány, M., Baumli, P., \& Kaptay, G. (2017). The influence of the phosphorous content and heat treatment on the nanomicro-structure, thickness and micro-hardness of electroless Ni-P coatings on steel. Applied Surface Science, 423, 160169. https://doi.org/10.1016/j.apsusc.2017.06.168

Doğan, F., Duru, E., Uysal, M., Akbulut, H., \& Aslan, S. (2020). Investigation of mechanical and tribological characteristics of ni-b coatıngs deposited on steel. Journal of Boron, 6(1), 209 215. https://doi.org/10.30728/boron.762584

Doğan, F., Uysal, M., Algül, H., Duru, E., Akbulut, H., \& Aslan, S. (2020). Optimization of pulsed electro co-deposition for Ni-B-TiN composites and the variation of tribological and corrosion behaviors. Surface and Coatings Technology, 400(June), 126209 https://doi.org/10.1016/j.surfcoat.2020.126209

Duru, E., Doğan, F., Uysal, M., Akbulut, H., \& Aslan, S. (2021). Optimization of Ni-B coating bath and effect of DMAB concentration on hardness and wear. Surfaces and Interfaces, 22(June 2020), 100880. https://doi.org/10.1016/j.surfin.2020.100880

Feng, L., Zhang, Y., Wen, C., L1, S., L1, J., Cheng, D., Ba1, J., Cu1, Q., \& Zhang, L. (2021). Effect of initial deposition behavior on properties of electroless Ni-P coating on ZK60 and ME20 magnesium alloys. Transactions of Nonferrous Metals Society of China, 31(8), 2307-2322. https://doi.org/10.1016/S1003-6326(21)65656-X

Ger, M.-D., \& Hwang, B. J. (2002). Effect of surfactants on codeposition of PTFE particles with electroless Ni-P coating. Materials Chemistry and Physics, 76(1), 38-45. https://doi.org/10.1016/S0254-0584(01)00513-2

Gültekin, D., Duru, E., \& Akbulut, H. (2021). Improved wear behaviors of lead-free electroless $\mathrm{Ni}$ [sbnd]B and $\mathrm{Ni}-\mathrm{B} / \mathrm{CeO} 2$ composite coatings. Surface and Coatings Technology, 422(July). https://doi.org/10.1016/j.surfcoat.2021.127525

Haag, S., Burgard, M., \& Ernst, B. (2006). Pure nickel coating on a mesoporous alumina membrane: Preparation by electroless plating and characterization. Surface and Coatings Technology, 201(6), 2166-2173. https://doi.org/10.1016/j.surfcoat.2006.03.023

Huang, Y. S., Zeng, X. T., Annergren, I., \& Liu, F. M. (2003). Development of electroless NiP-PTFE-SiC composite coating. Surface and Coatings Technology, 167(2-3), 207211. https://doi.org/10.1016/S0257-8972(02)00899-X

Luo, H., Leitch, M., Zeng, H., \& Luo, J.-L. (2018). Characterization of microstructure and properties of electroless duplex Ni-W-P/Ni-P nano-ZrO2 composite coating. Materials Today Physics, 4, 36-42. https://doi.org/10.1016/j.mtphys.2018.03.001

Matik, U., \& Citak, R. (2011). Düşük Fosforlu Akımsız Ni - P Kaplanmış AISI 1020 Çeliğinin Adhesiv A şınma Karakteristikleri nin Incelenmesi. May.

Ramalho, A., \& Miranda, J. C. (2005). Friction and wear of electroless NiP and NiP+PTFE coatings. Wear, 259(7-12), 828-834. https://doi.org/10.1016/j.wear.2005.02.052

Sürdem, S., Eseroğlu, C., \& Çitak, R. (2019). A parametric study on the relationship between $\mathrm{NaBH} 4$ and tribological properties in the nickel-boron electroless depositions. Materials Research Express, 6(12), 125085. https://doi.org/10.1088/2053-1591/ab5beb

Ürdem, Ş., Duru, E., Algül, H., Uysal, M., \& Akbulut, H. (2021). Evaluation of high temperature tribological behavior of electroless deposited NiB-Al2O3 coating. Wear, 482483(May), 203960. https://doi.org/10.1016/j.wear.2021.203960

Uysal, M., Algül, H., Duru, E., Kahraman, Y., Alp, A., \& Akbulut, H. (2021). Tribological properties of $\mathrm{Ni}-\mathrm{W}-\mathrm{TiO} 2-\mathrm{GO}$ composites produced by ultrasonically-assisted pulse electro co-deposition. Surface and Coatings Technology, 410(February), 126942. https://doi.org/10.1016/j.surfcoat.2021.126942

Vitry, V., \& Delaunois, F. (2015). Nanostructured electroless nickel-boron coatings for wear resistance. In Anti-Abrasive Nanocoatings (pp. 157-199). Elsevier. https://doi.org/10.1016/B978-0-85709-211-3.00007-8

Yildiz, R. A., Genel, K., \& Gulmez, T. (2021). Effect of electroless Ni-B and Ni-W-B coatings on the corrosionfatigue behaviour of $7075 \mathrm{Al}$ alloy. International Journal of Fatigue, 144(July 2020), 106040. https://doi.org/10.1016/j.ijfatigue.2020.106040. 